\title{
THE NONLINEAR DYNAMICS OF DENSE ELECTRON BEAMS IN THE AUTORESONANCE LASER ACCELERATOR
}

\author{
A. LOEB \\ Center for Plasma Physics, Racah Institute of Physics, Hebrew University of Jerusalem, Jerusalem 91904, Israel \\ and Plasma Physics Department, Soreq Nuclear Research Center, Yavne 70600, Israel \\ and \\ L. FRIEDLAND \\ Center for Plasma Physics, Racah Institute of Physics, Hebrew University of Jerusalem, Jerusalem 91904, Israel \\ Received 4 March 1988; accepted for publication 25 March 1988 \\ Communicated by R.C. Davidson
}

\begin{abstract}
The axial evolution of the electromagnetic fields is solved self-consistently with the nonlinear dynamics of the electron beam in the autoresonance laser accelerator (ALA). A $50 \mathrm{kA} / \mathrm{cm}^{2}$ electron beam can be accelerated over $100 \mathrm{~m}$ from an initial energy of $25 \mathrm{MeV}$ to $4.6 \mathrm{GeV}$ by a $\mathrm{CO}_{2}$ laser with a peak initial intensity of $2.8 \times 10^{14} \mathrm{~W} / \mathrm{cm}^{2}$ along a $100 \mathrm{kG}$ guide magnetic field.
\end{abstract}

Different approaches to the acceleration of charged particles to high energies by high power lasers have received much attention recently [1]. Within the far field approach two schemes were suggested, namely, the autoresonance laser accelerator (ALA) [2-4] and the inverse free electron laser (IFEL) [5]. The ALA scheme is based on a self-sustained cyclotron resonance between the particles and a circularly (or a linearly) polarized laser radiation propagating along an axisymmetric guide magnetostatic field. The resonance condition is $\Omega^{\prime} / \gamma=k_{0} v_{z}-\omega_{0}^{\prime}$, where $k_{0}$ and $\omega_{0}^{\prime}$ are the laser wavevector and frequency, and $\gamma, v_{z}$ and $\Omega^{\prime} / \gamma$ are the relativistic factor, the axial velocity and the relativistic cyclotron frequency of the particles. The detailed nonlinear single particle dynamics in the ALA fields configuration was analyzed recently [2]. It was shown that the acceleration of electrons to high energies with low radiation losses is possible in the ALA scheme for homogeneous electromagnetic fields. Therefore, it was suggested that the acceleration of high current beams might be feasible. It was also shown that the accelerated beam can be launched into the desired autoresonance regime through transition regions. The use of spon- taneously generated megagauss magnetic fields in laser produced plasmas for autoresonance acceleration was also discussed [3]. The simultaneous autoresonance acceleration of both electrons and positrons by a linearly polarized laser radiation presents a novel possibility of accelerating high current "quasi-neutral" beams to high energies [4].

In this Letter we shall discuss the collective acceleration of dense electron beams to high energies by high power lasers within the ALA scheme. Consider a circularly polarized laser radiation field with frequency $\omega_{0}^{\prime}$ propagating together with a cold electron beam of density $N$ along a homogeneous axial magnetic field $B_{0}$ in the $z$ direction. The acceleration of the electrons in the system is achieved by satisfying the cyclotron resonance condition at $z=0$ [2], and is accompanied by a self-consistent decrease of the laser field amplitude along the interaction region. We assume for the sake of simplicity a $1 \mathrm{D}$ model in which the system parameters have spatial dependence only along the $z$ axis. We define the following set of orthonormal base vectors, 
$\hat{\boldsymbol{e}}_{1}=-\hat{\boldsymbol{e}}_{x} \sin \theta+\hat{\boldsymbol{e}}_{y} \cos \theta$

$\hat{\boldsymbol{e}}_{2}=-\hat{\boldsymbol{e}}_{x} \cos \theta-\hat{\boldsymbol{e}}_{y} \sin \theta$,

$\hat{\boldsymbol{e}}_{3}=\hat{\boldsymbol{e}}_{z}$

where $\hat{\boldsymbol{e}}_{x}, \hat{\boldsymbol{e}}_{y}, \hat{\boldsymbol{e}}_{z}$ are unit vectors in the $x, y, z$ directions, respectively,

$\theta=\omega_{0}^{\prime}(z / c-t)$

and $c$ is the light velocity.

The electromagnetic fields $\boldsymbol{E}, \boldsymbol{B}$ as well as the density $N$ and velocity $v$ of the cold electron beam can be written as

$E=E_{1}(z) \hat{\boldsymbol{e}}_{1}+E_{2}(z) \hat{\boldsymbol{e}}_{2}=\boldsymbol{E}_{\perp}(z)$,

$\boldsymbol{B}=B_{1}(z) \hat{\boldsymbol{e}}_{1}+B_{2}(z) \hat{\boldsymbol{e}}_{2}+B_{3}(z) \hat{\boldsymbol{e}}_{3}$,

$V=V_{1}(z) \hat{\boldsymbol{e}}_{1}+V_{2}(z) \hat{\boldsymbol{e}}_{2}+V_{3}(z) \hat{\boldsymbol{e}}_{3}$

$N=N(z)$

The electrostatic field has been neglected in eq. (3), assuming that this field have small effect on the electrons dynamics compared to that of the intense laser electromagnetic fields at frequency $\omega_{0}^{\prime}$. As far as the radial effects are considered, this assumption is justified according to the Poisson equation if the electron beam current $J$ satisfies [2]

$J(k A) \ll 8 \omega_{0}^{\prime} R_{\mathrm{b}} \alpha / c$,

where $R_{\mathrm{b}}$ is the beam radius and $\alpha=e\left|E_{\perp}\right| / m \omega_{0}^{\prime} c$. Furthermore, the space charge effects can be cancelled in the simultaneous acceleration of electrons and positrons by using a linearly polarized laser radiation [4].

Since the frequency $\omega_{0}^{\prime}$ was already included in the basis vectors $\left\{\hat{\boldsymbol{e}}_{i}\right\}_{i=1,2,3}$ we shall assume that any variable in eqs. (3) $-(6)$ satisfies

$\left|\psi^{-1} \mathrm{~d} \psi / \mathrm{d} z\right| \ll \omega_{0}^{\prime} / c$,

where $\psi \equiv\left\{E_{i}, B_{i}, V_{i}, N\right\}_{i=1,2,3}$.

The Maxwell equations yield

$c \hat{\boldsymbol{e}}_{3} \times \partial \boldsymbol{B}_{\perp} / \partial z=\partial \boldsymbol{E}_{\perp} / \partial t-4 \pi e N \boldsymbol{V}_{\perp}$,

$-c \hat{e}_{3} \times \partial E_{\perp} / \partial z=\partial B_{\perp} / \partial t$,

$\partial B_{3} / \partial z=0$

where $\psi_{\perp}=\psi_{1} \hat{e}_{1}+\psi_{2} \hat{e}_{2}$. According to eq. (11),

$B_{3}=B_{0}=$ const and the wave equation is derived from eqs. (9) and (10),

$c^{2} \frac{\partial^{2} \boldsymbol{E}_{\perp}}{\partial z^{2}}=\frac{\partial^{2} \boldsymbol{E}_{\perp}}{\partial t^{2}}-4 \pi e N \frac{\partial \boldsymbol{V}_{\perp}}{\partial t}$.

By using the identities

$\frac{\partial \hat{\boldsymbol{e}}_{1}}{\partial t}=-\omega_{0}^{\prime} \hat{\boldsymbol{e}}_{2}, \quad \frac{\partial \hat{\boldsymbol{e}}_{2}}{\partial t}=\omega_{0}^{\prime} \hat{\boldsymbol{e}}_{1}$,

$\frac{\partial \hat{\boldsymbol{e}}_{1}}{\partial z}=\frac{\omega_{0}^{\prime}}{c} \hat{\boldsymbol{e}}_{2}, \quad \frac{\partial \hat{\boldsymbol{e}}_{2}}{\partial z}=-\frac{\omega_{0}^{\prime}}{c} \hat{\boldsymbol{e}}_{1}$,

we obtain in components

$\frac{\mathrm{d}^{2} E_{1}}{\mathrm{~d} z^{2}}=\frac{2 \omega_{0}^{\prime}}{c} \frac{\mathrm{d} E_{2}}{\mathrm{~d} z}-\frac{\omega_{0}^{\prime}}{c^{2}} 4 \pi e N V_{2}$,

$\frac{\mathrm{d}^{2} E_{2}}{\mathrm{~d} z^{2}}=-\frac{2 \omega_{0}^{\prime}}{c} \frac{\mathrm{d} E_{1}}{\mathrm{~d} z}+\frac{\omega_{0}^{\prime}}{c^{2}} 4 \pi e N V_{1}$.

For $N \rightarrow 0$ eqs. (15) and (16) yield the trivial solution $E_{i}=$ const $(i=1,2)$, which was assumed in previous discussions of the single particle dynamics in the ALA scheme [2-4]. In accordance with condition (8) we neglect the left-hand side of eqs. (15) and (16) so that

$\frac{\mathrm{d} E_{j}}{\mathrm{~d} z}=\frac{2 \pi e N}{c} V_{j}, \quad j=1,2$.

Assuming condition (8), eq. (10) yields

$B_{1}=\frac{\mathcal{c}}{\omega_{0}^{\prime}}\left(\frac{\mathrm{d} E_{1}}{\mathrm{~d} z}-\frac{\omega_{0}^{\prime}}{\mathcal{c}} E_{2}\right)=\frac{2 \pi e N}{\omega_{0}^{\prime}} V_{1}-E_{2}$,

$B_{2}=\frac{c}{\omega_{0}^{\prime}}\left(\frac{\mathrm{d} E_{2}}{\mathrm{~d} z}+\frac{\omega_{0}^{\prime}}{c} E_{1}\right)=\frac{2 \pi e N}{\omega_{0}^{\prime}} V_{2}+E_{1}$.

The continuity equation for the electron beam,

$\frac{\partial N}{\partial t}+\frac{\partial}{\partial z}\left(N V_{3}\right)=0$,

gives

$N V_{3}=N_{0} V_{30}=$ const .

The electrons momentum equation is given by

$\left(\frac{\partial}{\partial t}+V_{3} \frac{\partial}{\partial z}\right)(\gamma \boldsymbol{V})=-\frac{e}{m}\left(\frac{\boldsymbol{v}}{c} \times \boldsymbol{B}+\boldsymbol{E}\right)$,

where $\gamma=\left(1-v^{2} / c^{2}\right)^{-1 / 2}$ and the radiation losses are neglected [2]. The last equation and eqs. (17)-(21) written in components can be combined to give the 
following set of seven first order ordinary differential equations:

$$
\begin{aligned}
\dot{u}_{1} & =\frac{\omega_{0}}{\gamma}\left(\alpha_{1}\left(u_{1}^{2}+u_{3}-1\right)+\alpha_{2} u_{1} u_{2}-\frac{\Omega_{0}}{\omega_{0}} u_{2}\right. \\
& \left.-\frac{\Omega_{0}}{\omega_{0}} u_{2}-\left(1-u_{3}\right) \gamma u_{2}+\frac{N}{2 N_{\mathrm{c}}} u_{2} u_{3}\right), \\
\dot{u}_{2} & =\frac{\omega_{0}}{\gamma}\left(\alpha_{1} u_{1} u_{2}+\alpha_{2}\left(u_{2}^{2}+u_{3}-1\right)+\frac{\Omega_{0}}{\omega_{0}} u_{1}\right. \\
& \left.+\left(1-u_{3}\right) \gamma u_{1}-\frac{N}{2 N_{\mathrm{c}}} u_{2} u_{3}\right), \\
u_{3} & =\frac{\omega_{0}}{\gamma}\left(\alpha_{1} u_{1}+\alpha_{2} u_{2}\right)\left(u_{3}-1\right), \\
\dot{N} & =-N \dot{u}_{3} / u_{3}, \\
\dot{\gamma}= & -\omega_{0}\left(\alpha_{1} u_{1}+\alpha_{2} u_{2}\right), \\
\dot{\alpha}_{1} & =\left(N / 2 N_{\mathrm{c}}\right) \omega_{0} u_{1} u_{3}, \\
\dot{\alpha}_{2} & =\left(N / 2 N_{\mathrm{c}}\right) \omega_{0} u_{2} u_{3},
\end{aligned}
$$

where $\dot{\psi} \equiv c^{-1} \partial \psi / \partial t, \omega_{0}=\omega_{0}^{\prime} / c, u=v / c, \Omega_{0}=e B_{0} /$ $m c^{2}, \quad \gamma=\left(1-u_{1}^{2}-u_{2}^{2}-u_{3}^{2}\right)^{-1 / 2}, \alpha=e E / m c \omega_{0}^{\prime}$ and $N_{\mathrm{c}}=\left(m \omega_{0}^{\prime 2} / 4 \pi e^{2}\right)$ is the critical plasma density for the frequency $\omega_{0}^{\prime}$. Eqs. (23)-(29) describe the evolution of the electromagnetic fields in the system coupled to the nonlinear dynamics of the electron beam. These equations were solved numerically for different examples. It should be noted that the peak initial intensity of the laser radiation is related to $\alpha_{10}$ via the expression, $I_{0}=\left(2.8 \times 10^{18} \mathrm{~W} / \mathrm{cm}^{2}\right)\left(\alpha_{10} /\right.$ $\left.\lambda_{0}[\mu \mathrm{m}]\right)^{2}$, where $\lambda_{0}=2 \pi / \omega_{0}$.

Figs. 1 and 2 describe two examples of an efficient conversion of electromagnetic energy into the energy of the electron beam. These figures show the axial dependence of the electrons energy and the electric field amplitude $\alpha_{1}$ assuming that at $z=0$ the cyclotron resonance condition: $\Omega_{0}=\omega_{0} \gamma_{0}\left(u_{30}-1\right)$ is satisfied and $\alpha_{20}=u_{10}=u_{20}=0$. Fig. 1 shows the acceleration of a cold electron beam with an initial energy of $1 \mathrm{MeV}$ and current density of $250 \mathrm{kA} / \mathrm{cm}^{2}$ by a circularly polarized laser radiation with $\lambda_{0}=0.5 \mathrm{~mm}$ and $I_{0}=1.1 \mathrm{GW} / \mathrm{cm}^{2}$, along a homogeneous axial magnetic field of $55 \mathrm{kG}$. In this case almost all of the electromagnetic flux is converted into kinetic energy flux of the electrons in $75 \mathrm{~cm}$. Fig. 2 shows the acceleration of a $50 \mathrm{kA} / \mathrm{cm}^{2}$ electron beam with an ini-

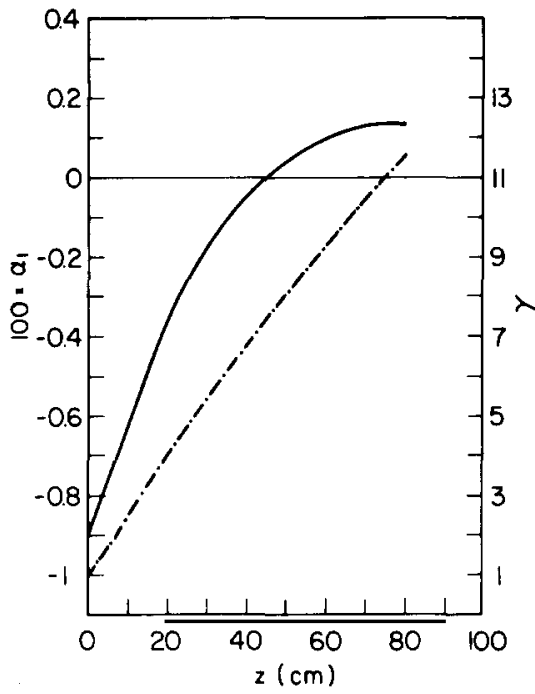

Fig. 1. Acceleration of an electron beam with $\gamma_{0}=2, N_{0}=5 \times 10^{10}$ $\mathrm{cm}^{-3}$ by a laser with $\omega_{0}=125 \mathrm{~cm}^{-1}, \alpha_{10}=-0.01$ along an axial magnetic field of $\Omega_{0}=-33 \mathrm{~cm}^{-1}$. The cyclotron resonance condition is satisfied at $z=0$ where $\alpha_{20}=u_{10}=u_{20}=0$. The spatial evolution of $\gamma$ (solid line) and $\alpha_{1}$ (dot-dashed) is shown.

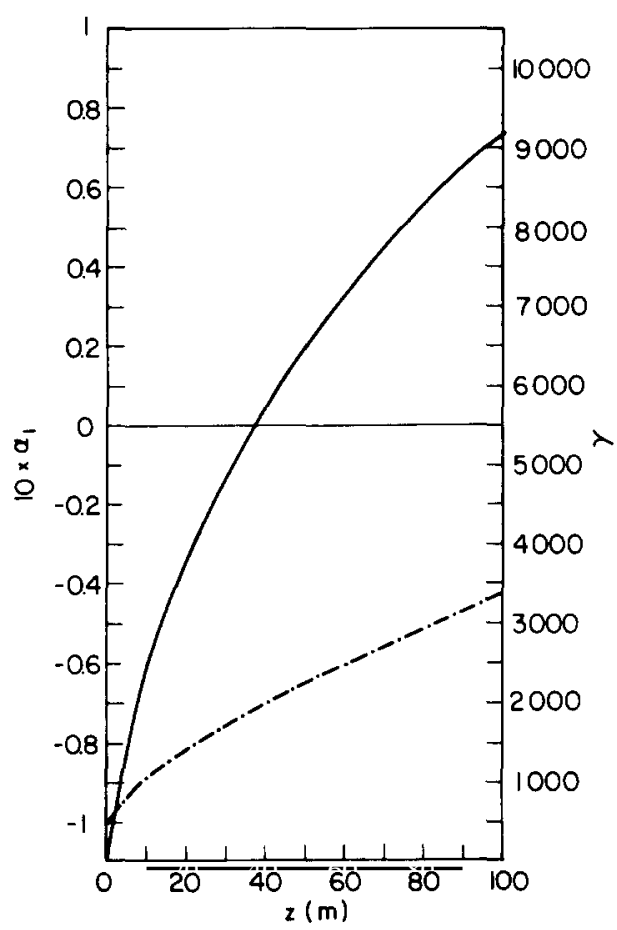

Fig. 2. The same as in fig. 1 for $\gamma_{0}=50, N_{0}=10^{13} \mathrm{~cm}^{-3}, \omega_{0}=6300$ $\mathrm{cm}^{-1}, \Omega_{0}=-63 \mathrm{~cm}^{-1}$ and $\alpha_{10}=-0.1$. 
tial energy of $25 \mathrm{MeV}$ by a $\mathrm{CO}_{2}$ laser $\left(\lambda_{0}=10 \mu \mathrm{m}\right)$ with a peak initial intensity of $2.8 \times 10^{14} \mathrm{~W} / \mathrm{cm}^{2}$ $\left(\alpha_{10}=-0.1\right)$ along a $100 \mathrm{kG}$ axial magnetic field. The electron beam is accelerated to an energy of 4.6 $\mathrm{GeV}$ and gains $82 \%$ of the electromagnetic power at a distance of $100 \mathrm{~m}$ where $\alpha_{1}=-0.042$ and $\alpha_{2}=-0.007$. It should be noted that the guidance of a high intensity laser beam over large distances is a crucial problem common to all the schemes in the far field approach for particles acceleration to high energies [1]. Alternative methods to the intensity-limited waveguides [ 1 ] are, for example, arrays of lenses, plasma fibers [3] or electron beam guidance [6]. In the context of the ALA scheme, this issue requires detailed discussion and will be considered elsewhere.

In conclusion, it was shown that dense electron beams can be accelerated efficiently to high energies, applying the autoresonance laser acceleration concept. Collective autoresonance acceleration of electrons or positions might also occur in astrophysical regions, such as pulsars, where strong magnetostatic fields and electromagnetic waves coexist.

\section{References}

[1] P.J. Channel, ed., Laser acceleration of particles, AIP Conf. Proc. No. 91 (AIP, New York, 1982);

The generation of high fields for particle acceleration to very high energies, Proc. CAS-ECFA-INFN Workshop, Frascati, Italy (CERN, Geneva, 1985);

C. Joshi and T. Katsouleas, eds., Laser acceleration of particles, AIP Conf. Proc. No. 130 (AIP, New York, 1986).

[2] A. Loeb and L. Friedland, Phys. Rev. A 33 (1986) 1828.

[3] A. Loeb and S. Eliezer, Phys. Rev. Lett. 56 (1986) 2252.

[4] A. Loeb, L. Friedland and S. Eliezer, Phys. Rev. A 35 (1987) 1692.

[5] C. Pellegrini, in: Laser acceleration of particles, AIP Conf. Proc. No. 91, ed. P.J. Channel (AIP, New York, 1982) pp. 138-153.

[6] E.T. Scharlemann, A.M. Sessler and J.S. Whurtele, Phys. Rev. Lett. 54 (1985) 1925. 